activity was acknowledged. Subsequently, main phases of the process were identified and described as subprocesses. The software used enabled linking between subprocess descriptions which made it possible to build up an overall picture of the process.

Lessons for Guideline Developers, Adaptors, Implementers, and/ or Users Visualising the overall picture of the process enables understanding of responsibilities of different performers in chronological order. Explicit process descriptions increase transparency, facilitate future process development, and help to maintain the rigorous guideline standards.

\section{P088 EXPLORING POSSIBILITIES FOR INTEGRATION OF RESULTS OF RESEARCH IN PATIENT PREFERENCES IN GUIDELINE DEVELOPMENT: PRELIMINARY RESULTS OF INTERVIEWS WITH SEVERAL STAKEHOLDERS.}

${ }^{1,2} \mathrm{C}$ Utens, ${ }^{1,2} \mathrm{M}$ Joore, ${ }^{3} \mathrm{~T}$ van der Weijden, ${ }^{1,2} \mathrm{C}$ Dirksen. ${ }^{1} \mathrm{Clinical}$ and Medical Technology Assessment, Maastricht University Medical Centre, Maastricht, The Netherlands; ${ }^{2}$ CAPHRI, School for Public Health and Primary Care, Maastricht University, Maastricht, The Netherlands, ${ }^{3}$ CAPHRI, School for Public Health and Primary Care, Department of General Practice, Maastricht, The Netherlands

\section{0:1136/bmjqs-2013-002293.154}

Background There is full awareness of the urge to integrate patient perspectives in guidelines. Active patient participation in guideline development is advocated, the passive use of research results on patient preferences is rather limited.

Objectives To explore ideas and opinions regarding potential barriers and facilitators for integrating research results on patient preferences in guideline development.

Methods Eight interviews were held with patient representatives, guideline developers, policy-makers and researchers. Interviews were semi-structured along three themes: definition of patient preferences; consideration of research on patient preferences in guideline development and aspects of obtaining patient preferences through research.

Results Most interviewees defined preferences broadly, using terms as ideas, values, wishes, needs, expectations and experiences. Others described preferences exclusively as comparative judgments. Interviewees had difficulties reflecting on considering patient preferences by using research results, instead of active participation. Although the general increasing focus on patient participation facilitates the use of research results, many barriers were mentioned: relevance of collective preference for individual decision-making; focus of evidence-based medicine on "hard evidence"; lack of reliable and valid data; unclear how to integrate research results into the development procedure.. Patient- and professional organisations often generate own evidence, with unclear scientific character.

Discussion The results show which issues are important and need further clearance. Interviewees define patient preferences differently, do not believe in using such research results or do not know how to do it.

Implications for Guideline Developers/Users Several issues need to be addressed to facilitate the integration of research results on patient preferences in guideline development.

\section{P089 CONSTRAINTS FOR CLINICAL GUIDELINE IMPLEMENTATION IN MONGOLIAN PRIMARY HEALTH CARE FACILITIES}

${ }^{1} \mathrm{~N}$ Sumberzul, ${ }^{2} \mathrm{E}$ Maximenco, ${ }^{2} \mathrm{~S}$ Ouynbileg, ${ }^{3} \mathrm{~A}$ Munkhtaivan, ${ }^{4} \mathrm{P}$ Jousilahti. ${ }^{1}$ Health Science University of Mongolia (HSUM), Ulaanbaatar, Mongolia; ${ }^{2}$ EPOS Health Management,
Ulaanbaatar, Mongolia; ${ }^{3}$ MCA Mongolia Health Project, Ulaanbaatar, Mongolia; ${ }^{4}$ National Institute for Health and Welfare (THL), Helsinki, Finland

\section{0:1136/bmjqs-2013-002293.155}

Background The goal of the Millennium Challenge Account Mongolia (MCA-Mongolia) Health Project is to reduce morbidity and mortality due to Non-Communicable Diseases (NCD) through extensive training of health staff, development of clinical guidelines and provision of equipment and other material resources. Four clinical guidelines were developed within the framework of the project: hypertension, type 2 diabetes, and breast and cervical cancers.

Objectives Facility Based Impact Study (FBIS) in 2010 gathered information on the capacity of health facilities to provide NCD services prior the project, and to assess the quality of services. Multi-stage stratified (urban and rural) sampling was used to select 194 primary health care facilities, and 730 individual respondents - representing different health worker categories were selected within the facilities. The quantity and quality of NCD related services were assessed based on five factors; (1) human resources, (2) NCD screening activities, (3) availability of standards and guidelines, (4) health education materials, and (5) equipment and supplies.

Results Only $10 \%$ of the facilities met the defined requirements for 'high quality' in the provision of NCD services, 38\% met the level of 'middle quality', $28 \%$ of facilities met the 'minimum level', and $24 \%$ were classified as facilities not meeting basic requirements and categorised as below the minimum quality level. Conclusions At least half of the health facilities need a marked improvement, and for one quarter the need is urgent. Insufficient training and time, and lack of materials were main barriers for effective NCD prevention and control.

\section{P092 DOES THE ADDITION OF SYMBOLS MAKE GUIDELINES RECOMMENDATIONS CLEARER? RESULTS FROM AN ONLINE SURVEY}

A Nast, S Rosumeck, A Jacobs, B Sporbeck. Dermatology, Charité - Universitätsmedizin Berlin, Berlin, Germany

\section{0:1136/bmjqs-2013-002293.156}

Background A recent study has shown that the perceived meaning of wordings in recommendations such as "should" or "must" can vary among guideline users. In addition to the wordings, many guidelines use graphic symbols such as arrows or smileys to support their recommendations.

Objectives To determine whether such symbols influence the perceived meaning of the recommendations or may help to reduce variation in the perception of the meaning between different guidelines' users.

Methods With the help of an online-survey, using a visual analogue scale (0-100), participating physicians from different specialties were asked to express their perceived levels of obligation when confronted with different guidelines recommendations in combination with different symbols.

Results 269 physicians participated, the addition of a "single arrow" or "double arrow" to the recommendation did not lead to relevant changes in the perceived obligation expressed by the recommendation (median: soll/shall: 83 vs. 87; "sollte/ should": 77 vs. 78 ). In comparison to the prior study, variations in the interpretation of typical guideline wordings were not reduced if symbols were used additionally. 